\title{
Funktionelle Körperbeschwerden sind Alltag
}

\author{
Niklaus Egloffa, Kyrill Schwegler ${ }^{a}$, Martin grosse Holtforth $^{a, b}$ \\ ${ }^{a}$ Kompetenzbereich für Psychosomatische Medizin, Universitätsklinik für Neurologie, Universitätsspital Inselspital, Bern; \\ b Institut für Psychologie, Universität Bern, Bern
}

Sowohl beim Grundversorger als auch bei der Spezialistin sind funktionelle Körperbeschwerden ein sehr häufiger Konsultationsgrund. Definitionsgemäss entziehen sich funktionelle Störungen einer biomorphologischen Befunderfassung auf Organebene. Die Symptome sind dennoch real und lassen sich heute im Rahmen übergeordneter Wahrnehmungs- und Steuerungsprozesse physiologisch verstehen. Die adäquate Kommunikation und Erklärung funktioneller Störungen ist häufig entscheidend für die Therapiemotivation des Betroffenen.

\section{Häufigkeit und Entstehung funktioneller Symptome}

Ungefähr jeder zweite bis fünfte Patient in der Grundversorgung leidet an funktionellen Beeinträchtigungen seiner Gesundheit [1]. Der Begriff funktionell wird häufig synonym mit somatoform oder psychosomatisch angewendet. Diese Begriffe überlappen bedeutungsmässig stark, implizieren aber unterschiedliche Krankheits-

Tabelle 1: Körpersymptome, die typischerweise auch funktionell bedingt sein können.

Mit Blick auf die üblichen Organerkrankungen zeigt sich, dass die Phänomenologie funktioneller Störungen stark mit den Leitsymptomen klassischer Organerkrankungen überlappt. Hier nicht aufgelistet sind somatische Erkrankungen (z.B. allergologische oder autoimmunologische Erkrankungen), die ebenfalls einer starken psychophysiologischen Modulation unterworfen sein können. Die nachfolgende Liste typischer funktioneller Symptome ist keinesfalls vollständig und ersetzt im Einzelfall nicht eine seriöse Differentialdiagnostik.

Schwindel, Benommenheit, Visusprobleme, Tinnitus, Fatigue, InsomKopf- und Hals- nie, Konzentrationsstörungen, Spannungskopfschmerzen, atypische organe Gesichtsschmerzen, epileptoide Anfallsstörungen, Räusperzwang, funktioneller Husten, Globussyndrom

Somatosensorische und motorische Ausfallstörungen, Bewegungsstö-

Extremitäten rungen, funktioneller Tremor, lokale, regionale oder generalisierte Weichteilschmerzen

Haut Juckreiz, Allodynie, Hyperhidrose, stressassoziierter Dermographismus, Erythrophobie

Herz-Kreislauf- Präkordialgien, atypische Thoraxschmerzen, Sinustachykardien, PalpiSystem tationen, hypertensive Entgleisungen, vasaovagale Synkopen

Atemorgane Tachypnoe, Hyperventilation, asthmaartige Krisen (meist im Rahmen Atemorgane von Panikattacken)

Schluckstörungen, nichtallergische und nicht-enzymatisch bedingte

Verdauungs- Nahrungsmittelunverträglichkeiten, Essstörungen, funktionelle Dys-

trakt pepsie, Völlegefühl, Obstipation, Diarrhoe, Reizdarmbeschwerden, funktionelle Abdominalschmerzen, Anismus

Urogenital- Chronische Becken- oder Genitalschmerzen, Miktionsstörungen, Dysbereich urie, Sexualfunktionsstörungen konzepte, was die Rolle psychologischer Faktoren bei der Krankheitsgenese und -aufrechterhaltung anbelangt. Die zielführende Betrachtungsperspektive zum physiologischen Symptomverständnis funktioneller Störungen liegt nicht auf der organpathologischen Strukturebene, sondern bei den Steuerungs- und Wahrnehmungsprozessen der Organe durch den Organismus. In beide Richtungen des Informationsflusses zwischen Organperipherie und dem empfindenden Gehirn gibt es vielfältige neuroimmunologische, neurovegetative und neuroendokrine Möglichkeiten, die zur Entstehung funktioneller Körperbeschwerden beitragen. Diese veränderten Steuerungs- und Wahrnehmungsprozesse stehen ihrerseits regelhaft in Bezug zu körperlichen Vorerfahrungen sowie psychobiographischen Prägungen, insbesondere derjenigen der übermässigen Stressexposition.

\section{Anspruchsvolle diagnostische Ausgangslage}

Phänomenologisch zeigen viele funktionelle Körperbeschwerden Symptomüberlappungen mit somatischen Erkrankungen, was die Differentialdiagnose anspruchsvoll macht (Tab. 1).

Detailcharakteristik, anatomische Verteilungsmuster, Modulationsfaktoren, Kombination mit anderen vegetativen oder hyperperzeptiven Symptomen, Dynamik und der biopsychosoziale Kontext erlauben die Diagnose einer funktionellen Störung jedoch meist mit hoher Wahrscheinlichkeit. Dabei ist anzumerken, dass sich strukturell und funktionell bedingte Symptome nicht ausschliessen, sondern häufig sogar gegenseitig 
bedingen. Bei vielen Schmerzerkrankungen zum Beispiel findet sich ein somatisches Auslöseereignis oder ein somatischer Kernanteil. Die funktionellen Sekundärprobleme sind dann der Grund, weshalb eine einzig auf die primäre Läsion ausgerichtete Schmerztherapie ungenügend wirkt.

\begin{abstract}
Fallvignette
Ein 35-jähriger Fabrikangestellter erfährt im Anschluss an einen Arbeitsunfall mit Weichteilverletzung an der rechten Schulter eine Schmerzausweitung auf den gesamten rechten oberen Quadranten. Die vom Patienten erstellte Schmerzzeichnung deutet zunächst auf ein myofasziales Verteilmuster unter Einbezug der Musculi trapezius, sternokleidomastoideus und occipitofrontalis hin. In der klinischen Untersuchung findet sich ein generell sehr angespannter Patient mit deutlichen nuchalen Myogelosen und einem ausgeprägter Dermographismus ruber. Schlafstörungen, Konzentrationsprobleme, Appetitminderung und Verdauungsbeschwerden weisen ebenfalls auf eine erhöhte Stressreaktion hin. Die zunächst wirksamen NSAR hatten ihren Effekt weitgehend verloren. Die aktuellen Symptome und Befunde sind nicht mehr mit der primären Läsion zu erklären, sondern weisen auf eine Schmerzdysregulationsstörung hin.
\end{abstract}

\section{Hyperperzeption}

Anatomische Basis für funktionelle Störungen ist das Nervensystem mit seinen peripheren, zentralen und autonomen Anteilen. Frühere Terminologien verwendeten dementsprechend auch die Begriffe nervöse Leiden oder Organ-Neurosen. Im Gegensatz zu klassisch neurologischen Erkrankungen sind bei funktionellen Störungen die Nerven zytomorphologisch intakt. Die Veränderungen betreffen also lediglich die Funktionalität der Nerven, was mit einer alterierten Physiologie auf Ebene der Rezeptoren sowie der Synapsen einhergeht. So sind bei funktionellen Körperschmerzen sehr häufig die Reizschwellen für Druck und Schmerz erniedrigt [2]. Dieser Aspekt der Hypersensibilität wurde unter dem Begriff der «Central Sensitivity Syndromes» präzise herausgearbeitet [3]. Vorerkrankungen, Dauerstress oder Traumata können die Sensibilisierung des Schmerzperzeptionssystems begünstigen, was zum wichtigen Arbeitsbegriff der stressinduzierten Hyperalgesie geführt hat [4].

\section{Fallvignette}

Die Frau des 35-jährigen Fabrikangestellten meint, ihr Mann sei in letzter Zeit viel empfindlicher und reizbarer geworden. Die Weichteilschmerzen gehen klinisch tatsächlich einher mit einer schmerzhaften Drucküberempfindlichkeit (Allodynie). Die algometrische Untersuchung weist ebenfalls auf eine erhöhte Schmerzempfindlichkeit (Hyperalgesie) hin. Der Patient erwähnt zudem eine Lärm- und Lichtüberempfindlichkeit, wie er sie von früheren Migränebeschwerden her kannte. Auch sei der vorbestehende Tinnitus deutlich lauter geworden. Die Frau bemerkt, dass der Patient sich schon vor dem Umfall etwas verändert hätte, seit er vornehmlich in der
Nachtschicht gearbeitet hätte. Auf die Nachtschicht angesprochen meint sie, dass er ihr damit ermögliche, nachmittags als Raumreinigerin arbeiten zu können. Er würde dann am Nachmittag auf die Kinder und die kranke Schwiegermutter aufpassen.

\section{Affektiv-kognitive Modulation des Symptomempfindens}

Unangenehme Körperempfindungen lösen stets eine affektive sowie eine kognitive Reaktion aus. So verursacht Schmerz regelhaft Angst und Besorgnis. Die Art dieser affektiv-kognitiven Wertung moduliert das Ausmass des subjektiven Leidens stark und stellt daher einen eigenständigen Ansatzpunkt für therapeutische Interventionen dar.

Komorbide Angst- und Depressionserkrankungen liegen bei Patientinnen und Patienten mit anhaltenden funktionellen Beschwerden gehäuft vor. Ist ein Patient generell in einer dysphorischen, verängstigten oder gekränkten Verfassung, wird dadurch die aversive Dimension des funktionellen Körperleidens oftmals noch verstärkt. ICD-11 propagiert für den Aspekt des übermässigen, präokkupierten Reagierens neu eine eigenständige Diagnose, die sogenannte somatische Belastungsstörung. ICD-10 beschritt zuvor den umgekehrten, quasi «zentrifugalen» Weg: Eine psychische Problematik führt über Somatisierungsvorgänge zu Körpersymptomen. In der klinischen Praxis kommen beide Wege sowohl alleine wie auch in Kombination vor. Fakt ist, dass Patientinnen und Patienten mit schwerwiegenden funktionellen Symptomstörungen gehäuft an psychischen Begleitbeschwerden leiden. Der Kausalitätsbezug zum funktionellen Körpersymptom ist aber komplex und kaum je unidirektional. So kann beispielsweise die frühe unsichere Bindungserfahrung Ursache einer anhaltend veränderten Stressphysiologie sein, Verhaltensschwierigkeiten mit sich bringen und psychische Folgen haben, die sich wiederum auf Perzeption und Regulation der Körperfunktionen auswirken.

\section{Fallvignette}

Der Patient vermutet, dass bei der Schulterverletzung etwas «kaputt gegangen» sei. Am liebsten würde er die Schulter "reparieren» lassen, um dann wieder arbeiten können. Er hätte nun deswegen schon drei Spezialisten aufgesucht. Der Orthopäde hätte gesagt, er hätte eine Depression und keine Schulterkrankheit. Er habe Angst, dass wenn man ihn nicht erst nähme, er tatsächlich noch eine Depression bekomme. Er wisse, was eine Depression sei. Seine Mutter hätte, seit er sich erinnern könne, immer wieder Depressionen gehabt. Er hoffe, das habe nicht auf inn abgefärbt. Er sei wegen der Krankheit der Mutter mehrheitlich bei den Grosseltern aufgewachsen.

\section{Dissoziatives Reagieren auf Symptome}

Übersteigen Körpersymptome oder emotionale Spannungen das «lebbare» Ausmass, kommen zusätzlich die 
Mechanismen der Dissoziation und der Konversion zum Zuge. Unter Dissoziation versteht man zentralnervöse Vorgänge, die zu einem Abspalten oder Ausschalten von Bewusstseinsinhalten oder körperlichen Empfindungen führt. In Bezug auf Körpersymptome führt dies mitunter zu einer Art Autoanästhesie, mit Sensibilitätsabschwächung oder Sensibilitätsverlust, bis hin zu einer Art Auto-Narkose mit vorübergehender Bewusstseinseintrübung. Diese Art von neurofunktionellen Ausfällen wird regelhaft bei schwer traumatisierten Patienten beobachtet; Leichtere Formen solch dissoziativer neurofunktioneller Störungen kommen aber auch bei weniger dramatischen Konstellationen vor.

Fallvignette

Auf den Arbeitsunfall angesprochen, berichtet der Patient, dass er noch heute häufig daran denken müsse. Während eines Nachtschichtdienstes hätte sich damals ein Metallstück des Deckenkrans gelöst und sei auf ihn herunter gedonnert. In den ersten Wochen nach dem Unfall hätte er oft davon geträumt. Er könne noch heute kaum in die Arbeitshalle gehen, weil er immer wieder vermute, es könnte sich etwas Ähnliches wiederholen. Das Schlimmste sei gewesen, dass er zunächst blutend am Boden gelegen hätte und es niemand bemerkte. Er hätte vermutlich dabei immer wieder das $\mathrm{Be}-$ wusstsein verloren. Noch heute, wenn er starke Schmerzen habe, schlafe der rechte Arm ein und der Hinterkopf fühle sich dann wie taub an.

\section{Hilfestellungen für die Praxis}

Obwohl funktionelle Körpersymptomstörungen sehr häufig sind, hat die Medizin noch keinen routinemässigen Umgang damit gefunden. Je intensiver man sich
Tabelle 2: Basisinformationen für Patienten mit funktionellen Beschwerden.

1. Symptome ohne nachweisbare körperliche Schädigung sind sehr häufig. Jeder Mensch kennt solche Symptome. Sie können grundsätzlich jedes Organsystem betreffen.

2. Diese Symptome sind keine Einbildung, sondern absolut real.

3. Funktionelle Symptome lassen sich aufgrund körperlicher (vorab vegetativer) Steuerungsvorgänge und/oder durch eine verstärkte Reizübertragung im Nervensystem (z.B. Reizdarm, Reizmagen, Reizblase) verstehen.

4. Vielfach stehen die Symptome in Zusammenhang mit den Auswirkungen von gesundheitlichen Vorereignissen oder Stress. Stress wirkt sich immer auf den ganzen Menschen aus. empfiehlt sich ein ganzheitlicher Therapieansatz, der oftmals medikamentöse, physiotherapeutische und stressreduzierende Massnahmen umfasst.

6. Funktionelle Symptome sind grundsätzlich gutartiger Natur.
5. Für eine Symptomatik mit mehreren Bedingungsfaktoren mit funktionellen Störungen befasst, umso deutlicher erkennt man, dass funktionelle Störungen nicht einfach nur Ausschlussdiagnosen sind, sondern eine eigene Positiv-Charakteristik haben. Die Zeichen vegetativer Dysregulation, somatosensorischer Hyperperzeption, emotional-kognitiver Präokkupation sowie einer biographischen Stress-Disposition müssen systematisch exploriert werden. Die Schweizerische Akademie für Psychosomatische Medizin (SAPPM) hat gemeinsam mit der Schweizerischen Gesellschaft zum Studium des Schmerzes (SGSS) dazu eine Positiv-Indikatoren-Liste für funktionelle (Schmerz-)Erkrankungen erstellt, die auch zur Begutachtung dieser Störungen dient [5]. Die von der Deutschen Arbeitsgemeinschaft der Wissenschaftlichen Medizinischen Fachgesellschaften (AWMF) regelmässig überarbeiten S-3 Leitlinien zu Funktionellen Körperbeschwerden enthalten konkrete therapeutische Wegleitungen. Im Rahmen einer multikausalen Symptomgenese ist auch der Therapieansatz multimodal zu planen [6].

Bei der Diagnosekommunikation muss das initiale ärztliche «Wording» mit Bedacht gewählt sein (Tab. 2). Wie in jeder komplexen Krankheitssituation ist auch hier eine tragfähige Arzt-Patienten-Beziehung für den Therapieverlauf von grossem Vorteil.

\section{Fallvignette}

Das vorliegende Fallbeispiel illustriert multiple pathophysiologische Mechanismen, die zu funktionellen Beschwerden führen könnten. Damit sich dieser Patient auf ein multimodalesTherapiekonzept einlassen konnte, musste er Gewissheit haben, dass die Behandelnden sein Leiden verstehen und nichts «verpassen» würden. Umgekehrt ist es auch für die Behandler notwendig, dass sich das Krankheitsverständnis einigermassen deckt. Der Einstieg hierfür erfolgte über folgenden verständnisfördernden SchmerzedukationsClip zu traumassoziierten Schmerzen: www.hklearning.net/CLIP/ Trauma.pdf. Damit wurden die Voraussetzungen geschaffen, dass der Patient sich auf eine physiotherapeutisch aktivierende, behaviourale, medikamentöse wie auch stützende psychologischeTherapie einlassen konnte. Gemeinsames Ziel war die Rückkehr an den Arbeitsplatz - allerdings in Zukunft mit nur noch reduzierter Nachtschichtpflicht.

\section{Literatur}

1 Haller H, Cramer H, Lauche R, Dobos G. Somatoforme Störungen und medizinisch unerklärbare Symptome in der Primärversorgung. Dtsch Arztebl Int. 2015;112(16): 279-87.

2 Egloff N, Cámara RJ, von Känel R, Klingler N, Marti E, Ferrari ML: Hypersensitivity and hyperalgesia in somatoform pain disorders. General Hospital Psychiatry 2014; 36; 284-290.

3 Yunus MB. Fibromyalgia and overlapping disorders: the unifying concept of central sensitivity syndromes. Semin Arthritis Rheum 2007; 36: 339-356

4 Jennings EM, Okine BN, Roche M, Finn DP. Stress-induced hyperalgesia. Prog Neurobiol 2014;121:1-18.

5 https://www.sappm.ch/ueber-uns/begutachtung

6 https://www.awmf.org/leitlinien/detail/ll/051-001.html 